\title{
Tuberous sclerosis complex with left ventricular noncompaction: a case description
}

\author{
Xiaoying Zhao ${ }^{1}$, Xinxiang Zhao ${ }^{1}$, Hua Wei $^{2}$, Fuwei Jin ${ }^{1}$ \\ ${ }^{1}$ Department of Radiology, The Second Affiliated Hospital of Kunming Medical University, Kunming, China; ${ }^{2}$ Department of Information, The \\ Second Affiliated Hospital of Kunming Medical University, Kunming, China \\ Correspondence to: Xinxiang Zhao, PhD. Department of Radiology, The Second Affiliated Hospital of Kunming Medical University, Kunming 650101, \\ China. Email: zhaoxinxiang06@126.com.
}

Submitted Aug 22, 2021. Accepted for publication Jan 20, 2022.

doi: $10.21037 /$ qims-21-834

View this article at: https://dx.doi.org/10.21037/qims-21-834

\section{Introduction}

Tuberous sclerosis complex (TSC) is a rare genetic multisystem disorder that affects roughly 1 in 10,000 people worldwide (1). TSC is considered a protean disease because TSC can occur in multiple organs of the body and the symptoms vary widely. According to the TSC Diagnostic Consensus released in 2012, there are two independent diagnosis criteria: clinical diagnosis and genetic diagnosis. Since genetic tests may not be able to identify mutations in up to $25 \%$ of TSC patients, and normal genetic test results do not rule out TSC, clinical diagnosis is the main method (2).

Cardiac manifestations of TSC are mostly rhabdomyoma. There have been no reports of TSC patients with left ventricular noncompaction (LVNC). This is the first report of an adult female diagnosed with TSC with the complication of LVNC.

\section{Case report}

A 34-year-old female was admitted to the hospital due to cough and expectoration for 2 months. The sputum was yellow and viscous. Shoulder and chest pain were reported during coughing. Hoarseness and feebleness were also described. Physical examination after admission revealed shagreen patches on her cranial region and back. Blood examination results suggested mild anemia, increased thrombocytosis, and high sensitivity of C-reactive protein. The patient had a healthy daughter and denied similar familial history.

\section{Imaging findings}

Due to cough symptoms and surgical history of appendicitis, the patient underwent chest and abdominal computed tomography (CT). Chest CT images showed bilateral scattered small ill- and well-defined nodules, ranging in size from 4-6 mm (Figure 1). Multiple variablyshaped sclerotic bone foci was observed in the sternum, thoracic vertebra, lumbar pyramidal and pedicle, rib, right humerus, and pelvis. The diameters varied from 3-10 mm (Figure 2). Abdominal CT displayed multiple slightly highdensity nodules in bilateral kidneys and the liver. The initial assessment was a tumor with metastasis. To explore the primary lesion, brain and abdominal magnetic resonance imaging (MRI) was conducted.

Brain MRI showed multiple cortical lesions in frontal and parietal lobes which were best depicted on fluidattenuated inversion-recovery (FLAIR) image (Figure $3 A$ ), and enhanced subependymal nodules along the left lateral ventricular surface were found (Figure 3B). In abdominal MRI images, bilateral hypointensity nodules in the renal parenchyma were showed on T2 weighted imaging (T2WI) (Figure 4). In opposed-phase chemical shift sequences, the signal of these lesions was not reduced. Moderate enhancement were observed after the contrast agent injection. A hepatic lump with a diameter of $23 \mathrm{~mm}$ was discovered in Couinaud II, which appeared as mild hypointense on T1 weighted imaging (T1WI) and mildly hyperintense on T2WI. The degree of enhancement was slightly higher than in normal liver parenchyma (Figure 5). 


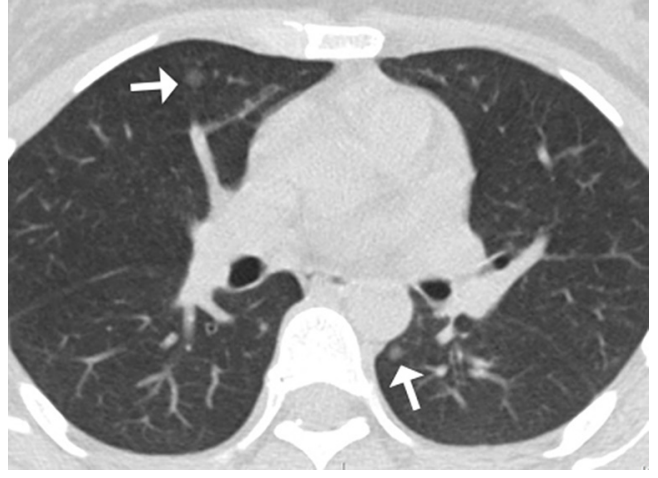

Figure 1 Chest plain CT scan (lung window) showed multiple ground glass-like density nodules (arrows) characteristic of MMPHs in bilateral lungs. CT, computerized tomography; MMPH, multifocal micronodular pneumocyte hyperplasia.

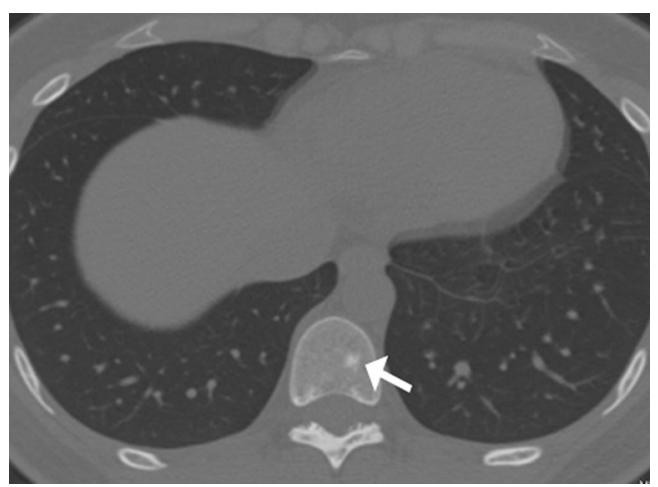

Figure 2 Chest plain CT scan (bone window), a skeletal sclerosis nodule (arrow) was found in thoracic vertebrae. CT, computerized tomography.

Together, these symptoms were considered as TSC multifocal micronodular pneumocyte hyperplasia (MMPH) in bilateral pulmonary, cortical dysplasia, subependymal nodules, multiple renal angiomyolipomas (AMLs) lacking fat, hepatic AML, and shagreen patch. We also found skeletal sclerosis nodules, which are common in TSC patients. In order to determine the cardiac involvement, cardiovascular magnetic resonance (CMR) was conducted, and LVNC of the lateral wall and apex was observed (Figure 6A,6B). The value of non-compact myocardium/compact myocardium (N/C) was $>2.3$, and the left ventricular ejection fraction (LVEF) decreased slightly to $46.2 \%$. Therefore, the diagnosis of the patient was TSC complicated with LVNC.

After the disease was defined, multidisciplinary consultations were conducted. The patient's symptoms
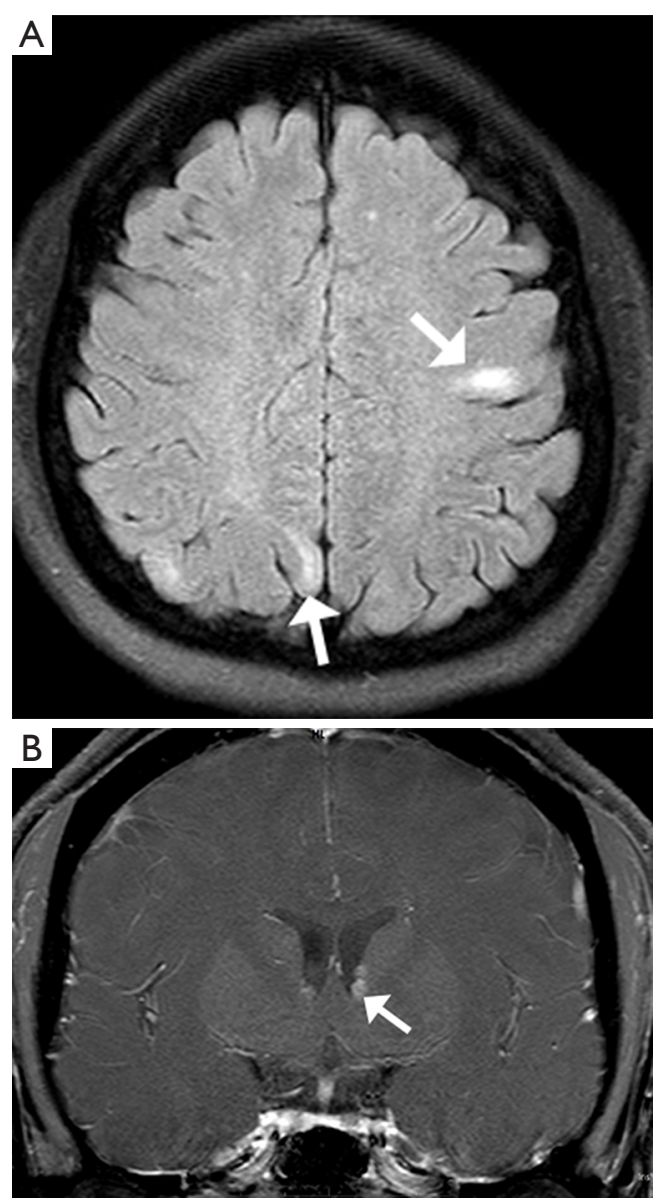

Figure 3 The brain MRI of the patient showed the typical cortical dysplasia and subependymal nodules in TSC. Axial MRI FLAIR showed cortical dysplasia manifested as irregular and slightly higher signal lesions (arrows) within the brain parenchyma involving the frontal and parietal lobes (A). Coronal gadolinium enhancement image showed enhanced subependymal nodules (arrow) (B). MRI, magnetic resonance imaging; TSC, tuberous sclerosis complex; FLAIR, fluid-attenuated inversion-recovery.

improved after receiving anti-inflammatory and antitussive therapy. Considering the patient's willingness and mild symptoms, physicians recommended a close follow-up but did not administer sirolimus medication. At the 6-month follow-up, the patient's condition was stable, and the lesions showed no progression. All procedures performed in studies were in accordance with the ethical standards of the institutional research committee and with the Helsinki Declaration (as revised in 2013). Written informed consent was obtained from the patient for publication of this case report and accompanying images. A copy of the written 


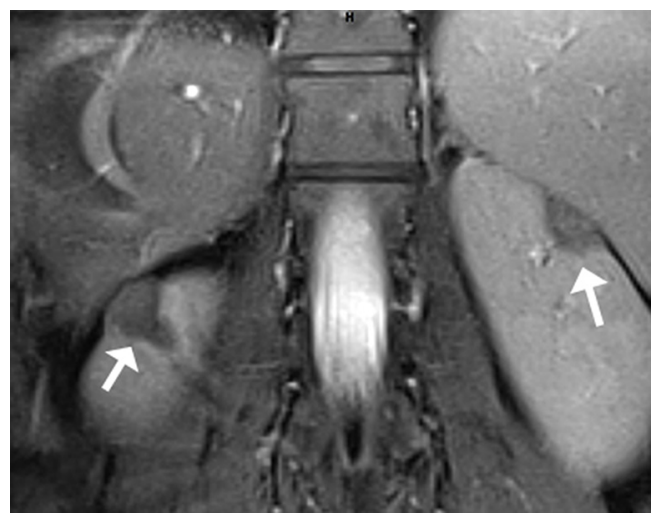

Figure 4 Coronal MRI T2WI showed that the lesions (arrows) protrude from the bilateral kidneys and outside the renal contours. MRI, magnetic resonance imaging; T2WI, T2 weighted imaging.

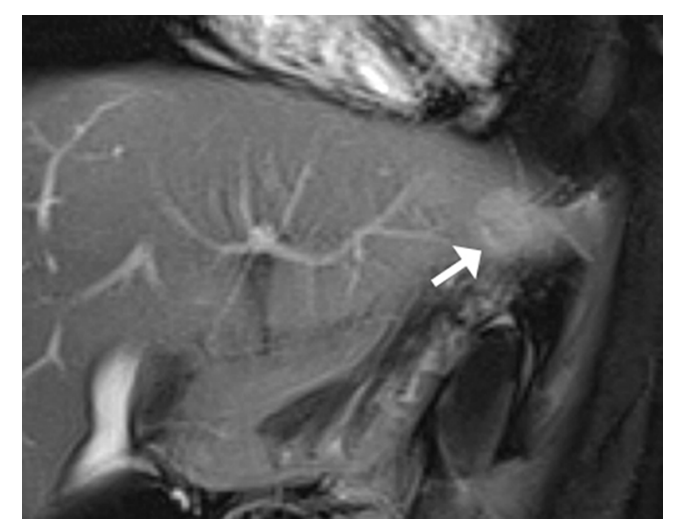

Figure 5 Round and slightly long signal foci (arrow) in the left lateral lobe of the liver were observed in coronal MRI T2WI. MRI, magnetic resonance imaging; T2WI, T2 weighted imaging.

consent is available for review by the editorial office of this journal.

\section{Discussion}

Most TSC manifestations appear during early childhood, except pulmonary and renal disease. Women diagnosed with TSC in adulthood usually present renal AMLs but without family history (3). This case was consistent with that described in the literature.

TSC-related skin lesions are prevalent and easily observed, including shagreen patches, facial angiofibromas, fibrous cephalic plaque, and ungual fibromas. Retinal hamartoma and retinal pigment deficiency spots are classified into dermatological manifestations (4). The brain is most
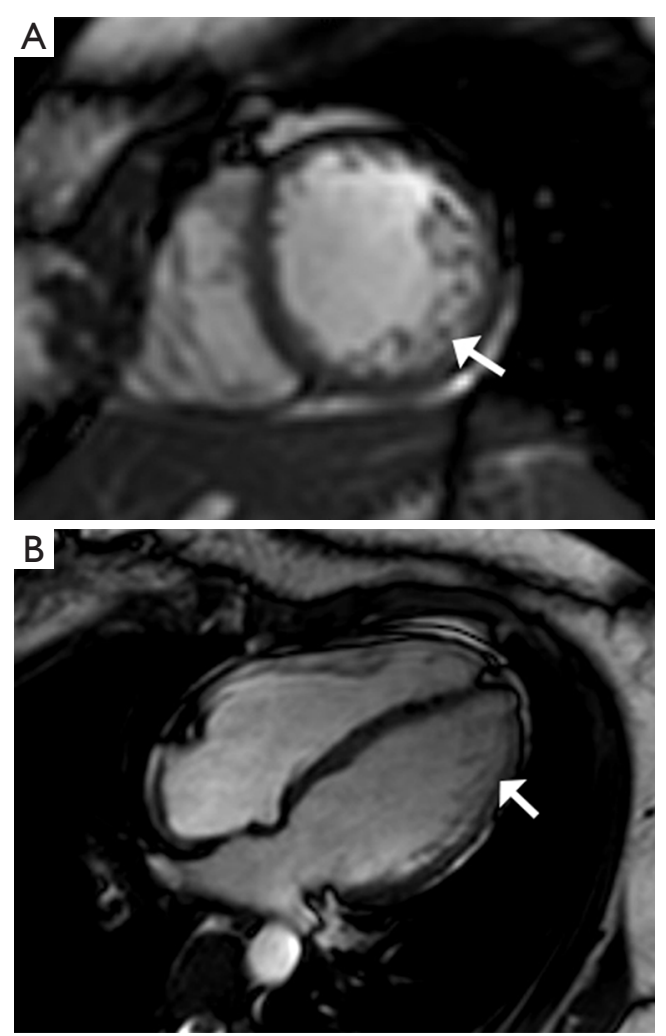

Figure 6 CMR manifestations of the patient met the diagnostic criteria of LVNC. In the CMR short-axis (A) and four-chamber images (B), the distance from the lateral wall epicardium to the medial edge of the trabeculae increased while presenting a two-layer structure (arrow). The ratio of N/C was >2.3. CMR, cardiovascular magnetic resonance; LVNC, left ventricular noncompaction; N/C, non-compact myocardium/compact myocardium.

commonly involved in TSC. In this case, cortical dysplasia and subependymal nodules were detected. Lungs with MMPHs can observed in TSC patients with an incidence rate of $28-71 \%$. MMPH is manifested as a solid nodule or ground-glass opacity (5). The most common renal manifestations of TSC patients are AMLs, occurring in roughly $75 \%$ of patients (6). This case was characterized by AMLs in bilateral kidneys and the liver. Another remarkable feature of this case was skeletal manifestations that have been barely reported. The diagnostic sensitivity of skeletal sclerosis nodules to TSC was $89 \%$, and specificity was $97 \%$ (7).

Cardiac rhabdomyoma is common in TSC patients, while other cardiac manifestations-myocardial fat deposition and dilated cardiomyopathy, for example, have low possibility of occurrence $(8,9)$. However, TSC patients with LVNC have 
not been reported previously. LVNC is a rare congenital developmental disorder characterized by a gross disorder of trabecular structure, a deep recess between trabeculae, and a ratio of N/C $>2.3$ in end-diastolic. Determining the cause of LVNC remains controversial, and many gene mutations linked to the condition have not yet been substantiated. Patients with LVNC carries a high risk of malignant arrhythmia, thromboembolic phenomenon and left ventricular dysfunction (10). The case we reported had decreased LVEF (46.2\%), but no cardiac symptoms appeared.

The essence of TSC is a disorder of cell migration, proliferation, and differentiation. Mutations in TSC 1 or TSC 2 genes cause overactivation of the mammalian target of rapamycin (mTOR), resulting in abnormal cell proliferation and cardiac rhabdomyoma formation. mTOR inhibitors such as sirolimus can treat cardiac rhabdomyoma associated with TSC (11). LVNC is a genetically heterogeneous disease caused by the cessation of normal myocardial compaction in the embryonic period. It can occur in isolation or in association with other disorders and can also be a part of other genetic syndromes. In 2015, Muhammad described a large Bedouin family with severe recessive LVNC and found that autophagy flux was impaired in LVNC (12). In 2021, Al Kindi demonstrated the potential role of autophagy as a mechanism for genesis and regression of cardiac rhabdomyoma in TSC (13). Herein, we assumed a genetic association between the two disease processes. However, due to the limited number of previous papers, future research in this field is necessary. Some possibilities as to why no cases of TSC with LVNC have previously been reported before are as follows: first, echocardiography has been the preferred method of cardiac examination due to its low cost and efficiency, although it is possible that LVNC may be missed on echocardiography (14). Second, LVNC may be relatively rare in TSC. Thus, further CMR study in TSC is needed.

In conclusion, this report is the first case of TSC with LVNC, which improves our understanding of TSC and provides some inspiration for finding a link between the two diseases.

\section{Acknowledgments}

Funding: None.

\section{Footnote}

Conflicts of Interest: All authors have completed the ICMJE uniform disclosure form (available at https://qims. amegroups.com/article/view/10.21037/qims-21-834/coif). The authors have no conflicts of interest to declare.

Ethical Statement: The authors are accountable for all aspects of the work in ensuring that questions related to the accuracy or integrity of any part of the work are appropriately investigated and resolved. All procedures performed in studies were in accordance with the ethical standards of the institutional research committee and with the Helsinki Declaration (as revised in 2013). Written informed consent was obtained from the patient for publication of this case report and accompanying images. A copy of the written consent is available for review by the editorial office of this journal.

Open Access Statement: This is an Open Access article distributed in accordance with the Creative Commons Attribution-NonCommercial-NoDerivs 4.0 International License (CC BY-NC-ND 4.0), which permits the noncommercial replication and distribution of the article with the strict proviso that no changes or edits are made and the original work is properly cited (including links to both the formal publication through the relevant DOI and the license). See: https://creativecommons.org/licenses/by-nc-nd/4.0/.

\section{References}

1. Bishay VL, Crino PB, Wein AJ, Malkowicz SB, Trerotola SO, Soulen MC, Stavropoulos SW. Embolization of giant renal angiomyolipomas: technique and results. J Vasc Interv Radiol 2010;21:67-72.

2. Northrup H, Krueger DA; International Tuberous Sclerosis Complex Consensus Group. Tuberous sclerosis complex diagnostic criteria update: recommendations of the 2012 Iinternational Tuberous Sclerosis Complex Consensus Conference. Pediatr Neurol 2013;49:243-54.

3. Seibert D, Hong CH, Takeuchi F, Olsen C, Hathaway O, Moss J, Darling TN. Recognition of tuberous sclerosis in adult women: delayed presentation with life-threatening consequences. Ann Intern Med 2011;154:806-13, W-294.

4. Ebrahimi-Fakhari D, Meyer S, Vogt T, Pföhler C, Müller CSL. Dermatological manifestations of tuberous sclerosis complex (TSC). J Dtsch Dermatol Ges 2017;15:695-700.

5. Gupta N, Henske EP. Pulmonary manifestations in tuberous sclerosis complex. Am J Med Genet C Semin Med Genet 2018;178:326-37.

6. Bissler JJ, Kingswood JC. Renal angiomyolipomata. 
Kidney Int 2004;66:924-34.

7. Avila NA, Dwyer AJ, Rabel A, Darling T, Hong $\mathrm{CH}$, Moss J. CT of sclerotic bone lesions: imaging features differentiating tuberous sclerosis complex with lymphangioleiomyomatosis from sporadic lymphangioleiomymatosis. Radiology 2010;254:851-7.

8. Koyama M, Yano T, Kikuchi K, Miura T. Everolimusresponsive dilated cardiomyopathy in tuberous sclerosis. Eur Heart J 2015;36:2338.

9. Tresoldi S, Munari A, Di Leo G, Pompili G, Magistrelli P, Secchi F, La Briola F, Canevini MP, Cornalba G, Sardanelli F. Myocardial Fatty Foci in Adult Patients with Tuberous Sclerosis Complex: Association with Gene Mutation and Multiorgan Involvement. Radiology 2015;277:398-405.

10. Aung N, Doimo S, Ricci F, Sanghvi MM, Pedrosa C, Woodbridge SP, Al-Balah A, Zemrak F, Khanji MY, Munroe PB, Naci H, Petersen SE. Prognostic Significance of Left Ventricular Noncompaction: Systematic Review and Meta-Analysis of Observational Studies. Circ Cardiovasc Imaging 2020;13:e009712.

11. Chen XQ, Wang YY, Zhang MN, Lu Q, Pang LY, Liu LY,

Cite this article as: Zhao X, Zhao X, Wei H, Jin F. Tuberous sclerosis complex with left ventricular noncompaction: a case description. Quant Imaging Med Surg 2022;12(5):2995-2999. doi: 10.21037/qims-21-834
Li YF, Zou LP. Sirolimus Can Increase the Disappearance Rate of Cardiac Rhabdomyomas Associated with Tuberous Sclerosis: A Prospective Cohort and Self-Controlled Case Series Study. J Pediatr 2021;233:150-5.e4.

12. Muhammad E, Levitas A, Singh SR, Braiman A, Ofir R, Etzion S, Sheffield VC, Etzion Y, Carrier L, Parvari R. PLEKHM2 mutation leads to abnormal localization of lysosomes, impaired autophagy flux and associates with recessive dilated cardiomyopathy and left ventricular noncompaction. Hum Mol Genet 2015;24:7227-40.

13. Al Kindi HN, Ibrahim AM, Roshdy M, Abdelghany BS, Yehia D, Masoud AN, Simry W, Aguib Y, Yacoub MH. Clinical, cellular, and molecular characterisation of cardiac rhabdomyoma in tuberous sclerosis. Cardiol Young 2021;31:1297-305.

14. Fonseca AC, Marto JP, Pimenta D, Guimarães T, Alves PN, Inácio N, Viana-Baptista M, Pinho E Melo T, Pinto FJ, Ferro JM, Almeida AG. Undetermined stroke genesis and hidden cardiomyopathies determined by cardiac magnetic resonance. Neurology 2020;94:e107-13. 